\title{
Drop Suspension Final Dosage Form
}

National Cancer Institute

\section{Source}

National Cancer Institute. Drop Suspension Final Dosage Form. NCI Thesaurus. Code C68997.

A suspension prepared immediately prior to dispensing or administration in a drop-wise fashion. 